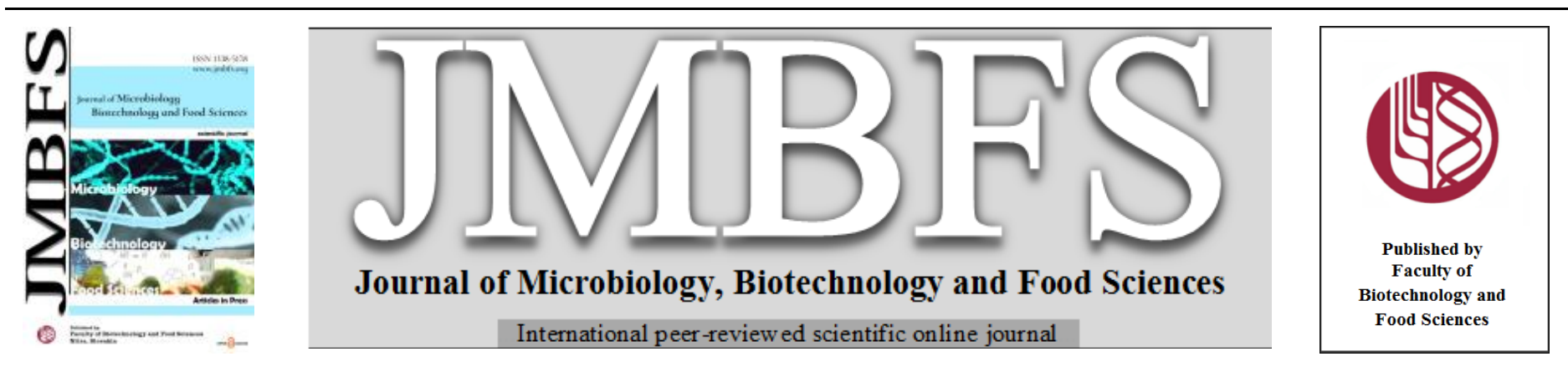

\title{
CARRIER DEVELOPMENT FOR PGPR-BIOFERTILIZER AND ITS EXPLOITATION UNDER CADMIUM AND LEAD STRESSED CONDITION
}

\author{
Amit Kumar Pal, Balaram Sarkar and Chandan Sengupta*
}

$\operatorname{Address(es):~}$

Microbiology Research Laboratory, Department of Botany, University of Kalyani, Kalyani 741235, West Bengal, India.

*Corresponding author: chandansenguptaku@gmail.com

https://doi.org/10.55251/jmbfs.2506

\section{ARTICLE INFO}

Received 31. 12. 2019

Revised 13. 1. 2022

Accepted 18. 1.2022

Published 1. 6. 2022

Regular article OPEN $\partial_{\text {AcCESS }}$

\begin{abstract}
Worldwide overpopulation needs progressive crop yield creating environmental pollution. Low cost and easily available biofertilizer development is the best alternative for a sustainable agricultural system. In this study different agricultural waste such as wheat bran, sesame bran and bagasse were used as a carrier for the isolated PGPR. Two most potent cadmium and lead tolerant PGPR were isolated from Indian agricultural soil and identified as Lysinibacillus varians strain KUBM17 (accession number MG976681) and Pseudomonas putida strain KUBM18 (accession number MG976684). PGPRs were inoculated in different possible combinations of carrier materials. The growth and survivability of PGPRs were checked with plate count technique frequently up to 120 days. The best carrier was selected for PGPR application on radish and gram plants to check their plant growth-promoting ability. Sesame bran showed higher CFU count $\left(7.8 \times 10^{6} / \mathrm{gm}\right)$ for the PGPRs from the early days. Whereas, wheat bran and bagasse individually showed slower but sustainable nutrient availability for both the PGPRs. Wheat bran, sesame bran and bagasse in 1:1:1 ratio produced decent performance throughout the time period. The carrier mixture as biofertilizer showed higher plant biomass, germination percentage, chlorophyll content and other growth parameters on radish and gram plant under cadmium and lead stress. Carrier grown Lysinibacillus varians and Pseudomonas putida were able to nullify the toxicity of cadmium or lead and significantly $(\mathrm{p}<0.05)$ enhanced almost all the plant growth parameters of both the plants. So, wheat bran, sesame bran and bagasse can be used for cheap PGPR-biofertilizer development.
\end{abstract}

Keywords: PGPR; $\mathrm{Cd}$ and $\mathrm{Pb}$ toxicity; carrier development; radish plant; gram plant

\section{INTRODUCTION}

Worldwide increasing population needs more and more crop yield for their existence. Industrialization and urbanization have posed to excessive land pollution especially with different heavy metals. Massive use of chemical fertilizer and pesticides to increase agricultural yield and to control pathogens are often effective but at the same time, causing adverse ecological, economic and social problems (Choure and. Dubey, 2012). Different agrochemicals, anthropogenic sources, urban waste and effluents from Ni-Cd battery, tannery and leather industry cause heavy metal contamination in agricultural system. In sustainable agricultural system bacterial fertilizers and bio-pesticides can be a good alternative for chemical fertilizer and chemical pesticides because fungal pathogens are prone to be chemical resistant easily (Prikryl and Vancura, 1980). On the other hand chemical compounds may be dangerous for other beneficial microorganisms. These bacteria that promote plant growth and yield are called as plant growthpromoting rhizobacteria (PGPR) (Ningthoujam et al., 2009). Several materials were used as carrier for developing biofertilizer like peat soil, coal, FYM, compost, charcoal, lignite, talc, cellulose powder, bagasse, teak leaf meal, sedge peat, press mud, coconut shell powder, (Tilak et al., 1979) fly ash etc. (Samra et al., 2003; Ajaya and Chhonkar, 2000). Materials, which make available nutrients and habitable micro-pore to the microorganisms especially for bacteria, are generally used as carrier.

Biofertilizers are the carrier-based inoculants which contain microorganisms of various specific capabilities. The usage of carrier materials for the biofertilizer as microbial inoculants is beneficial to protect the bacteria and its effectiveness (Fuentes-Ramirez and Caballero-Mellado, 2005; Ardakani et al., 2010). Among various types of carrier materials, peat is the most commonly used material due to its high moisture holding capacity and large surface area. Peat can support high number of microorganisms with long survivability. But it was reported to have toxic contaminants that affect the viability of bacteria (Bashan, 1998).

There are different agricultural bi-product such as rice hull (husk), sesame bran, bagasse, wheat bran etc. which are usually discarded after harvesting of the yeild or some time burned that are the main concern of air pollutionin India and other developing countries. The substrates rice husk, rice bran, wheat bran, maize bran, khesari bran, soybean bran, saw dust and mustard oilcake (MOC) were already studied for bio-fungicide production (Faruk et al., 2014).

In this study, three different types of agricultural waste were considered as carrier substrate such as sesame bran, wheat bran and Bagasse (Sugarcane waste). Bagasse is a rich source of carbohydrates (sucrose, fructose etc.) that can be utilized as nutrients of different bacteria (Dotaniya et al., 2016). Wheat bran contains almost all of the B-group vitamins: thiamine, riboflavin, niacin, pantothenic acid, pyridoxine, biotin, folates and different essential amino acids such as lysine, arginine glycine etc. (Fardet, 2010) with different carbohydrates. According to Ravindran and Jaiswal, (2016), by-products from different grains have high nutritional value. Sesame bran contains several minerals like potassium (4.6-5.3 g/kg), phosphorus (1.7-2.3 g/kg) magnesium (0.018-0.052 g/kg), calcium in high concentration (9.6-12.8 g/kg), amino acid like tryptophan and some carbohydrates (Alyemeni et al., 2011; Zouari et al., 2016).

In this study a proper carrier development for the isolated PGPR has been discussed. Moreover, carrier grown, $\mathrm{Cd}$ and $\mathrm{Pb}$ tolerant PGPRs were exploited on two plants such as radish and gram under heavy metal stressed condition.

\section{MATERIALS AND METHODS}

\section{Collection of Bacteria}

Two potent cadmium and lead tolerant plant growth promoting rhizobacteria were isolated from Indian agricultural soil by standard microbiological techniques. These bacteria were characterized and identified as Lysinibacillus varians (NCBI GenBank accession number MG976681) and Pseudomonas putida (NCBI GenBank accession number MG976684). Characterization and identification were previously published earlier (Pal and Sengupta, 2019). 


\section{Characterization of Bacteria}

The collected Cd and lead tolerant PGPR were characterized with morphological and biochemical properties such as amylase, catalase, methyl red, citrate utilization, gelatine hydrolysis, indole production, urease and different carbohydrate utilization ability by standard protocol (Pal and Sengupta, 2019). Plant growth promoting abilities such as IAA production, Phosphate solubilization, ammonia production, HCN production, nitrogen-fixing ability and siderophore production were determined (Pal and Sengupta, 2019).

\section{Carbohydrate utilization ability of PGPR}

Carbohydrate utilization efficiency was determined by inoculation of isolated bacteria in synthetic broth medium $\left(\mathrm{NH}_{4} \mathrm{Cl} 5.0 \mathrm{~g} ; \mathrm{K}_{2} \mathrm{HPO}_{4} 3.0 \mathrm{~g} ; \mathrm{Na}_{2} \mathrm{SO}_{4} 2.0 \mathrm{~g}\right.$ $\mathrm{KH}_{2} \mathrm{PO}_{4} 1.0 \mathrm{~g} ; \mathrm{NH}_{2} \mathrm{NO}_{3} 1.0 \mathrm{~g} ; \mathrm{MgSO}_{4}$. $7 \mathrm{H}_{2} \mathrm{O} 0.1 \mathrm{~g}$; distilled water 1 lit, $\mathrm{pH} 7.0$ 土 0.2 ) incorporated with anyone carbohydrate among Glucose, fructose, lactose, galactose, arabinose, cellobiose, dextrose, inositol, mannitol, maltose, raffinose and sucrose $(2.0 \mathrm{mg})$. After $48 \mathrm{~h}$ of incubation growth was measured at $600 \mathrm{~nm}$ (Pal and Sengupta, 2019).

\section{Carrier Formulation}

Different agricultural waste such as wheat bran, sesame bran and bagasse were used for the preparation of carrier. Selected PGPRs were inoculated in different combinations of sterile carrier. The growth and survivability of PGPRs were checked with typical microbiological plate count technique subsequently after 60 and 120 days from the inoculation.

The different experimental setups for the carrier development were as follows:

$\begin{array}{ll}\text { 1. } & \mathrm{WB}+\mathrm{B} 1 \\ 2 . & \mathrm{SB}+\mathrm{B} 1 \\ 3 . & \mathrm{Ba}+\mathrm{B} 1 \\ 4 . & \mathrm{WB}+\mathrm{SB}+\mathrm{B} 1 \\ 5 . & \mathrm{SB}+\mathrm{Ba}+\mathrm{B} 1 \\ 6 . & \mathrm{WB}+\mathrm{Ba}+\mathrm{B} 1 \\ 7 . & \mathrm{WB}+\mathrm{SB}+\mathrm{Ba}(1: 1: 1)+\mathrm{B} 1 \\ 1 . & \mathrm{WB}+\mathrm{B} 2 \\ 2 . & \mathrm{SB}+\mathrm{B} 2 \\ 3 . & \mathrm{B}+\mathrm{B} 2 \\ 4 . & \mathrm{WB}+\mathrm{SB}+\mathrm{B} 2 \\ 5 . & \mathrm{SB}+\mathrm{Ba}+\mathrm{B} 2 \\ 6 . & \mathrm{WB}+\mathrm{Ba}+\mathrm{B} 2 \\ 7 . & \mathrm{WB}+\mathrm{SB}+\mathrm{Ba}(1: 1: 1)+\mathrm{B} 2\end{array}$

N.B. WB- Wheat bran; SB- Sesame bran; Ba- Bagasse; B1- Lysinibacillus varians; B2Pseudomonas putida

\section{Exploitation of PGPR within carrier}

\section{Collection of Seeds}

Radish (Raphanus sativus) and Gram (Cicer arietinum) seeds were collected from the agricultural seed house Bidhan Chandra Krishi Viswavidyalaya, Kalyani, Nadia, W.B. India.

\section{Preparation of Soil}

Barren soil was collected, sieved and sterilized at $121^{\circ} \mathrm{C}$ and $15 \mathrm{lb}$ pressure for 1 hour for consecutive 3 days as performed previously by Pal, Chakraborty, and Sengupta (2018). Cadmium (10 ppm) and/or Lead (150 ppm) were determined on the basis of EC50 on seed germination, mixed with soil according to experimental design and left it for 1 week to stabilize.

\section{Experimental Design}

The complete experiment was conducted under controlled laboratory conditions Meanwhile, bacterial inoculums were prepared with best and efficient carrier concentration. The treatment sets were as follows:

$\begin{array}{ll}\text { 1. } & \text { Control } \\ \text { 2. } & \text { Cadmium }(\mathrm{Cd}) \\ \text { 3. } & \mathrm{Cd}+\mathrm{B} 1 \\ \text { 4. } & \mathrm{Cd}+\mathrm{B} 2 \\ \text { 5. } & \mathrm{Cd}+\mathrm{B} 1+\mathrm{B} 2 \\ \text { 6. } & \mathrm{Lead}(\mathrm{Pb}) \\ \text { 7. } & \mathrm{Pb}+\mathrm{B} 1 \\ 8 . & \mathrm{Pb}+\mathrm{B} 2 \\ \text { 9. } & \mathrm{Pb}+\mathrm{B} 1+\mathrm{B} 2 \\ \text { 10. } & \mathrm{Cd}+\mathrm{Pb} \\ \text { 11. } & \mathrm{Cd}+\mathrm{Pb}+\mathrm{B} 1 \\ \text { 12. } & \mathrm{Cd}+\mathrm{Pb}+\mathrm{B} 2 \\ \text { 13. } & \mathrm{Cd}+\mathrm{Pb}+\mathrm{B} 1+\mathrm{B} 2 \\ \mathrm{~B} 1=\text { KUBM } 17 \text { grown in carrier }\end{array}$

\section{$\mathrm{B} 2=\mathrm{KUBM} 18$ grown in carrier}

The healthy seeds were surface sterilized and placed in pot for germination and seedling growth. Respective bacterial inoculums prepared within the carrier were applied. Standard agricultural practices were taken and pots were displaced to minimize the position effects. $\mathrm{pH}$ of soil or carrier before PGPR inoculation and after 120 days of inoculation was measured by Jackson (1973).

\section{In vitro plant growth promotion experiment}

Germination percentage was calculated after 7 days of potting. Root length $(\mathrm{cm})$, Shoot length (cm.), Fresh weight (gm.), Dry weight (gm.), Vigor Index (AbdulBaki and Anderson, 1973), Relative water content and Chlorophyll content (mg/gm of tissue) (Porra et al. 1989) were recorded after 45 days of growth.

Vigour index $=$ Germination $(\%) \mathrm{x}$ Total seedling length $(\mathrm{cm}$.

Relative water content $(\%)=(\mathrm{FW}-\mathrm{DW} / \mathrm{TW}-\mathrm{DW}) \times 100$

Where FW = Fresh weight

DW $=$ Dry weight

TW $=$ Turgid weight

\section{Statistical analysis}

Standard error (SE) of all experimental data was anticipated from triplicates $(\mathrm{n}=$ 3 ) and represented in the respective table in 'value $\pm S E$ ' format. Differences between the experimental groups were intended by unpaired t-test. Difference between control and respective heavy metal treated groups were denoted by lower case alphabet ' $a$ '. Lower case alphabet ' $b$ ' indicates differences between heavy metal-treated groups and carrier grown PGPR inoculated heavy metal-treated groups. Asterisk mark (*) indicates a significant level.

\section{RESULTS AND DISCUSSION}

Two potent cadmium and lead tolerant PGPRs were isolated, characterized and identified by the authors. Biochemical characterization, carbohydrate utilization ability and plant growth promoting ability are being discussed in Table 1 .

For application of Lysinibacillus varians and Pseudomonas putida in agricultura field in commercial basis it need to develop a cheap and easily available carrier. WB, SB or Ba showed good CFU (colony forming unit) count of the PGPRs but different combinations of carriers showed very promising outcome (Table 2). WB and $\mathrm{Ba}$ individually gave slower but long term nutrient availability for both the PGPRs, whereas, the PGPRs utilized SB vigorously from the initial stage and showed highest CFU count at 60 days. In this study it revealed that the survival ability of Lysinibacillus varians and Pseudomonas putida were not just maintained but also increased in a 1:1 mixture of $\mathrm{WB}+\mathrm{SB}$ for at least 120 days. SB complimenting the nutrient availability of $\mathrm{WB}$ or $\mathrm{Ba}$ for the $\mathrm{CFU}$ counts from 60days to at least 120 days. Moreover, $\mathrm{WB}+\mathrm{SB}+\mathrm{Ba}$ in $1: 1: 1$ ratio represented a descent survivability of Lysinibacillus varians and Pseudomonas putida throughout the time period. On the basis of that result $\mathrm{WB}+\mathrm{SB}+\mathrm{Ba}$ can be commercially applied by the farmers as a carrier composition for biofertilizer production.

In vitro plant growth promotion experiment by carrier grown PGPR strains on radish and gram plant under cadmium and lead stress.

Application of cadmium and lead tolerant PGPRs as biofertilizers developed by using the best proportion of different carrier showed very promising results during the plant growth experiments. Heavy metal hyper-accumulator Radish and nonhyper-accumulator gram plant showed similar plant growth promotion induced by Lysinibacillus varians and Pseudomonas putida under cadmium and lead stress condition. Different plant growth parameter such as germination percentage, root length, shoot length, fresh weight, dry weight, chlorophyll content, vigor index and turgid weight for radish and gram plant are depicted in Table no 3 and 4 respectively. Cadmium, lead or combination of cadmium and lead significantly ( $p$ $<0.05$ ) reduced germination percentage, root length, shoot length, fresh weight, dry weight, chlorophyll content, vigor index and turgid weight in both radish and gram plant. Consortium of Lysinibacillus varians and Pseudomonas putida within developed carrier $(\mathrm{WB}+\mathrm{SB}+\mathrm{Ba})$ reduced the adverse effects of heavy metals and different growth parameters were increased significantly $(\mathrm{p}<0.05)$. Radish and gram plant showed promising growth enhancement with PGPR application than the non-inoculated $\mathrm{Cd}$ and/or $\mathrm{Pb}$ treated plants. As the developed carrier were applied in all the setups including control pots and the pots were interchanged in their positions, it can be assume that nutrients within the carrier and position effect are negligible among the treatments. While, the nutrients within the carrier material can be as attributed to extra benefit for the plant growth enhancement in the agricultural field. 
Table 1 Colony morphology, Gram nature, Biochemical characterization and PGP ability of the Bacterial isolates

\begin{tabular}{|c|c|c|c|c|c|}
\hline $\begin{array}{l}\text { Bacterial } \\
\text { isolates }\end{array}$ & $\begin{array}{l}\text { Minimal Inhibitory } \\
\text { Concentration of } \\
\text { cadmium and lead }\end{array}$ & $\begin{array}{l}\text { Colony Morphology \& } \\
\text { Gram nature }\end{array}$ & $\begin{array}{c}\text { Biochemical } \\
\text { characterization }\end{array}$ & $\begin{array}{c}\text { Carbohydrate utilization } \\
\text { ability }\end{array}$ & $\begin{array}{c}\text { Plant growth promoting } \\
\text { ability }\end{array}$ \\
\hline L. varians & $\begin{array}{l}150 \mathrm{ppm}(\mathrm{Cd}) \\
450 \mathrm{ppm}(\mathrm{Pb})\end{array}$ & $\begin{array}{l}\text { Creamy yellow, circular } \\
\text { with serrated margins, } \\
\text { opaque with a glossy } \\
\text { surface, Gram-positive, rod }\end{array}$ & $\begin{array}{l}\text { Catalase, methyl red, citrate } \\
\text { utilization tests positive and } \\
\text { amylase, gelatin hydrolysis, } \\
\text { indole production, urease } \\
\text { tests negative }\end{array}$ & $\begin{array}{l}\text { Glucose, Fructose, Arabinose, } \\
\text { Sucrose, Inositol, Manitol, } \\
\text { Maltose }\end{array}$ & $\begin{array}{l}\text { ammonia production, } \\
\text { IAA production }(20.43 \pm \\
0.318 \mu \mathrm{g} / \mathrm{ml}), \text { Nitrogen- } \\
\text { fixing ability, low } \\
\text { phosphate solubilization } \\
\text { and low siderophore } \\
\text { production positive }\end{array}$ \\
\hline
\end{tabular}

Table 2 Survivility of PGPR for different carrier development

\begin{tabular}{|c|c|c|c|c|}
\hline \multirow{3}{*}{ Different carrier $^{a}$} & \multicolumn{4}{|c|}{ Bacterial count (CFU/gm of carrier) ${ }^{b}$} \\
\hline & \multicolumn{2}{|c|}{ KUBM 17} & \multicolumn{2}{|c|}{ KUBM 18} \\
\hline & 60 days & 120 days & 60 days & 120 days \\
\hline WB & $0.56 \times 10^{6}$ & $15 \times 10^{9}$ & $0.92 \times 10^{6}$ & $19.9 \times 10^{9}$ \\
\hline SB & $7.8 \times 10^{6}$ & $9.7 \times 10^{9}$ & $13.52 \times 10^{6}$ & $10 \times 10^{9}$ \\
\hline $\mathrm{Ba}$ & $0.24 \times 10^{6}$ & $11.8 \times 10^{9}$ & $0.13 \times 10^{6}$ & $11 \times 10^{9}$ \\
\hline $\mathrm{WB}+\mathrm{SB}$ & $7.13 \times 10^{6}$ & $12 \times 10^{9}$ & $8.96 \times 10^{6}$ & $11.9 \times 10^{9}$ \\
\hline $\mathrm{SB}+\mathrm{Ba}$ & $8.21 \times 10^{6}$ & $7.86 \times 10^{9}$ & $14 \times 10^{6}$ & $10.8 \times 10^{9}$ \\
\hline $\mathrm{WB}+\mathrm{Ba}$ & $8.44 \times 10^{6}$ & $12.5 \times 10^{9}$ & $1.92 \times 10^{6}$ & $19.98 \times 10^{9}$ \\
\hline $\mathrm{WB}+\mathrm{SB}+\mathrm{Ba}$ & $7.6 \times 10^{6}$ & $13.1 \times 10^{9}$ & $9.4 \times 10^{6}$ & $12.8 \times 10^{9}$ \\
\hline
\end{tabular}

${ }^{\mathrm{a}}$ Different carrier was inoculated with broth culture of respective bacterial isolates (WB= Wheat bran, $\mathrm{SB}=$ Sesame bran, $\left.\mathrm{Ba}=\mathrm{Bagassi}\right)$

${ }^{\text {b }}$ Initial bacterial count was $\sim 10^{2} \mathrm{CFU} / \mathrm{gm}$ of carrier.

Table 3 In vitro plant growth promotion experiments by carrier grown PGPR strain on radish plant under cadmium and lead stress.

\begin{tabular}{|c|c|c|c|c|c|c|c|c|}
\hline Treatment & $\begin{array}{l}\text { Germination } \\
\text { percentage \% }\end{array}$ & Root length (cm) & Shoot length $(\mathbf{c m})$ & $\begin{array}{l}\text { Fresh weight } \\
\text { (gm) }\end{array}$ & Dry weight (gm) & Chl $(a+b)$ & Vigor index & $\begin{array}{l}\text { Turgid weight } \\
\text { (gm) }\end{array}$ \\
\hline Control & $83.33 \pm 0.0001$ & $4.94 \pm 0.508$ & $5.7 \pm 0.634$ & $0.44 \pm 0.092$ & $0.03 \pm 0.0061$ & $38.04 \pm 0.577$ & 474.98 & $0.4 \pm 0.021$ \\
\hline $\mathrm{Cd}$ & $87.5 \pm 2.78 \mathrm{a}^{* *}$ & $3.4 \pm 0.277 \mathrm{a}^{*}$ & $4.8 \pm 0.428 \mathrm{a}^{*}$ & $0.26 \pm 0.049 \mathrm{a}^{* *}$ & $0.02 \pm 0.0033 \mathrm{NS}$ & $30.25 \pm 0.203 \mathrm{a}^{* * *}$ & $420 \mathrm{a}^{*}$ & $0.24 \pm 0.022 \mathrm{a}^{* * * *}$ \\
\hline $\mathrm{Cd}+\mathrm{L}$. varians & $94.64 \pm 2.06 \mathrm{~b}^{* * *}$ & $6.43 \pm 0.262 \mathrm{~b}^{* * *}$ & $6 \pm 0.311 \mathrm{~b} * *$ & $0.59 \pm 0.068 \mathrm{~b}^{* * * *}$ & $0.04 \pm 0.0061 \mathrm{~b} * *$ & $37.03 \pm 0.289 \mathrm{~b}^{* * *}$ & $605.7 \mathrm{~b} * * *$ & $0.5 \pm 0.012 b^{* * *}$ \\
\hline $\mathrm{Cd}+$ P. putida & $91.67 \pm 2.06 b^{* * *}$ & $5.08 \pm 0.383 \mathrm{~b}^{* *}$ & $6.11 \pm 0.495 \mathrm{~b}^{* *}$ & $0.4 \pm 0.141 \mathrm{~b}^{* *}$ & $0.02 \pm 0.0043 \mathrm{NS}$ & $32.87 \pm 0.432 \mathrm{~b}^{* * * *}$ & $559.17 \mathrm{~b}^{* * * *}$ & $0.36 \pm 0.018 \mathrm{~b}^{* *}$ \\
\hline $\begin{array}{l}\text { Cd+ L. varians+ } \\
\text { P. putida }\end{array}$ & $96.43 \pm 2.38 b^{* * *}$ & $6.25 \pm 0.47 \mathrm{~b}^{* * *}$ & $6.38 \pm 0.55 \mathrm{~b}^{* *}$ & $1.06 \pm 0.222 \mathrm{~b}^{* * *}$ & $0.06 \pm 0.0104 \mathrm{~b}^{* * *}$ & $37.76 \pm 0.307 \mathrm{~b}^{* * *}$ & $617.15 \mathrm{~b}^{* * *}$ & $0.83 \pm 0.032 \mathrm{~b}^{* * * *}$ \\
\hline $\mathrm{Pb}$ & $55 \pm 5.02 \mathrm{a}^{* * *}$ & $4.01 \pm 0.404 \mathrm{a}^{*}$ & $4.35 \pm 0.379 \mathrm{~b}^{* *}$ & $0.31 \pm 0.055 \mathrm{a}^{*}$ & $0.03 \pm 0.0037 \mathrm{NS}$ & $24.94 \pm 0.58 \mathrm{a}^{* * *}$ & $330 \mathrm{a}^{* * * *}$ & $0.18 \pm 0.014 \mathrm{a}^{* * *}$ \\
\hline $\mathrm{Pb}+\mathrm{L}$. varians & $64.29 \pm 0.0001 \mathrm{~b}^{* * *}$ & $4.95 \pm 0.289 \mathrm{~b}$ & $5.35 \pm 0.148 \mathrm{~b}^{*}$ & $0.82 \pm 0.156 \mathrm{~b}^{* * *}$ & $0.05 \pm 0.0077 \mathrm{~b}^{* *}$ & $27.69 \pm 0.61 \mathrm{~b}^{*}$ & $447.16 b^{* * * *}$ & $0.55 \pm 0.028 \mathrm{~b}^{* * * *}$ \\
\hline $\mathrm{Pb}+\mathrm{P}$. putida & $66.86 \pm 7.04 \mathrm{~b}^{* * *}$ & $5.32 \pm 0.339 \mathrm{~b}^{*}$ & $4.88 \pm 0.33 \mathrm{NS}$ & $0.61 \pm 0.104 \mathrm{~b}^{* *}$ & $0.03 \pm 0.0046 \mathrm{NS}$ & $35.56 \pm 0.27 \mathrm{~b}^{* * *}$ & $427.61 \mathrm{~b} * * *$ & $0.35 \pm 0.004 \mathrm{~b}^{* * * *}$ \\
\hline $\begin{array}{l}\mathrm{Pb}+\mathrm{L} \text {. varians+ } \\
\text { P. putida }\end{array}$ & $76.78 \pm 7.8 \mathrm{~b}^{* * *}$ & $5.96 \pm 0.657 \mathrm{~b}^{* *}$ & $6.66 \pm 0.0237 \mathrm{~b}^{* * *}$ & $0.91 \pm 0.124 \mathrm{~b}^{* * *}$ & $0.09 \pm 0.0156 \mathrm{~b}^{* * *}$ & $33.83 \pm 0.81 \mathrm{~b}^{* * *}$ & $514.43 \mathrm{~b}^{* * *}$ & $0.73 \pm 0.035 \mathrm{~b}^{* * *}$ \\
\hline $\mathrm{Cd}+\mathrm{Pb}$ & $50 \pm 3.92 \mathrm{a}^{* * *}$ & $3.02 \pm 0.25 \mathrm{a}^{*}$ & $5.11 \pm 0.589 \mathrm{NS}$ & $0.46 \pm 0.049 \mathrm{NS}$ & $0.01 \pm 0.0046 \mathrm{a}^{*}$ & $19.96 \pm 0.244 \mathrm{a}^{* * *}$ & $260 \mathrm{a}^{* * * *}$ & $0.11 \pm 0.008 \mathrm{a}^{* * *}$ \\
\hline $\begin{array}{l}\mathrm{Cd}+\mathrm{Pb}+\mathrm{L} . \\
\text { varians }\end{array}$ & $57.14 \pm 0.0001 \mathrm{~b}^{* *}$ & $5.55 \pm 0.409 \mathrm{~b}^{* * *}$ & $6.2 \pm 0.433 \mathrm{~b}^{*}$ & $0.72 \pm 0.077 b^{* *}$ & $0.04 \pm 0.0023 \mathrm{NS}$ & $26.25 \pm 0.91 \mathrm{~b}^{* * *}$ & $388.55 \mathrm{~b} * * *$ & $0.43 \pm 0.017 \mathrm{~b}^{* * *}$ \\
\hline $\begin{array}{l}\mathrm{Cd}+\mathrm{Pb}+\mathrm{P} \\
\text { putida }\end{array}$ & $66.07 \pm 2.06 \mathrm{~b}^{* * *}$ & $5.01 \pm 0.512 b^{* *}$ & $5.45 \pm 0.286 \mathrm{NS}$ & $0.55 \pm 0.063 \mathrm{~b}^{*}$ & $0.03 \pm 0.0023 \mathrm{NS}$ & $25.09 \pm 0.56 \mathrm{~b}^{* * *}$ & $324.12 b^{* * *}$ & $0.33 \pm 0.024 \mathrm{~b}^{* * *}$ \\
\hline $\begin{array}{l}\mathrm{Cd}+\mathrm{Pb}+\mathrm{L} . \\
\text { varians }+\mathrm{P} \\
\text { putida }\end{array}$ & $58.93 \pm 8.5 \mathrm{~b}^{* *}$ & $7.66 \pm 0.795 \mathrm{~b}^{* * *}$ & $6.76 \pm 0.387 b^{* *}$ & $0.82 \pm 0.104 \mathrm{~b}^{* * *}$ & $0.06 \pm 0.0086 \mathrm{NS}$ & $25.72 \pm 0.399 \mathrm{~b}^{* * * *}$ & $429.45 \mathrm{~b}^{* * *}$ & $0.62 \pm 0.024 \mathrm{~b}^{* * *}$ \\
\hline
\end{tabular}


Table 4 In vitro plant growth promotion experiments by carrier grown PGPR strain on gram plant under cadmium and lead stress.

\begin{tabular}{|c|c|c|c|c|c|c|c|c|}
\hline Treatment & $\begin{array}{c}\text { Germination } \\
\text { percentage } \\
(\%)\end{array}$ & Root length (cm) & Shoot length $(\mathbf{c m})$ & $\begin{array}{l}\text { Fresh weight } \\
\text { (gm) }\end{array}$ & $\begin{array}{l}\text { Dry weight } \\
\text { (gm) }\end{array}$ & Chl $(a+b)$ & Vigor index & $\begin{array}{l}\text { Turgid weight } \\
\text { (gm) }\end{array}$ \\
\hline Control & $74 \pm 1.095$ & $6.9 \pm 0.463$ & $35.23 \pm 0.884$ & $1.02 \pm 0.108$ & $0.25 \pm 0.176$ & $38.04 \pm 0.5$ & 1850 & $0.42 \pm 0.012$ \\
\hline $\mathrm{Cd}$ & $54 \pm 0.57 \mathrm{a}^{* * *}$ & $5.5 \pm 0.294 \mathrm{a}^{* *}$ & $25.23 \pm 0.884 \mathrm{a}^{* * *}$ & $0.74 \pm 0.142 \mathrm{a}^{*}$ & $0.12 \pm 0.08 \mathrm{a}^{* *}$ & $25.09 \pm 0.5 \mathrm{a}^{* * *}$ & $1035.18 \mathrm{a}^{* * * *}$ & $0.23 \pm 0.031 \mathrm{a}^{* * *}$ \\
\hline $\mathrm{Cd}+\mathrm{L}$. varians & $90 \pm 0.547 \mathrm{~b}^{* * *}$ & $7.26 \pm 0.762 b^{* * *}$ & $29.66 \pm 2.245 \mathrm{~b}^{* *}$ & $1.17 \pm 0.06 \mathrm{~b}^{* *}$ & $0.29 \pm 0.01 \mathrm{~b}^{* *}$ & $27.03 \pm 0.3 \mathrm{~b}^{* *}$ & $2670 \mathrm{~b}^{* * * *}$ & $0.41 \pm 0.014 \mathrm{~b}^{* * *}$ \\
\hline $\mathrm{Cd}+\mathrm{P}$. putida & $68 \pm 1.341 \mathrm{~b}^{* *}$ & $6 \pm 0.935 b^{*}$ & $27 \pm 0.707 \mathrm{NS}$ & $1.04 \pm 0.89 \mathrm{~b}^{*}$ & $0.17 \pm 0.08 \mathrm{NS}$ & $32 . .87 \pm 0.21 \mathrm{~b}^{* * * *}$ & $1896 \mathrm{NS}$ & $0.36 \pm 0.033 \mathrm{~b}^{* *}$ \\
\hline $\begin{array}{l}\text { Cd+ L. varians+ } \\
\text { P. putida }\end{array}$ & $92 \pm 0.758 \mathrm{~b}^{* * *}$ & $7.76 \pm 0.601 \mathrm{~b} * * *$ & $29.56 \pm 1.493 \mathrm{~b}^{* *}$ & $1.16 \pm 0.1 \mathrm{~b}^{* *}$ & $0.36 \pm 0.01 \mathrm{~b}^{* * *}$ & $44.94 \pm 0.18 \mathrm{~b}^{* * *}$ & $2720 \mathrm{~b}^{* * * *}$ & $0.45 \pm 0.008 \mathrm{~b}^{* * *}$ \\
\hline $\mathrm{Pb}$ & $60 \pm 0.707 \mathrm{a}^{*}$ & $5.8 \pm 0.681 \mathrm{a}^{* *}$ & $29.3 \pm 4.893 \mathrm{a}^{* *}$ & $0.89 \pm 0.089 \mathrm{NS}$ & $0.11 \pm 0.01 \mathrm{a}^{* *}$ & $15.72 \pm 0.24 \mathrm{a}^{* * * *}$ & $1758 \mathrm{NS}$ & $0.33 \pm 0.026 \mathrm{a}^{* *}$ \\
\hline $\mathrm{Pb}+\mathrm{L}$. varians & $82 \pm 0.414 b^{* * *}$ & $7.3 \pm 0.696 b^{* *}$ & $38.46 \pm 1.728 \mathrm{~b}^{* *}$ & $1.26 \pm 0.08 \mathrm{~b}^{*}$ & $0.16 \pm 0.01 \mathrm{NS}$ & $19.96 \pm 0.51 b^{* * *}$ & $3154.54 \mathrm{~b}^{* * * *}$ & $0.49 \pm 0.057 b^{* * *}$ \\
\hline $\mathrm{Pb}+\mathrm{P}$. putida & $70 \pm 0.866 \mathrm{~b}^{* *}$ & $6.1 \pm 0.308 \mathrm{NS}$ & $35.76 \pm 4.745 b^{* *}$ & $1.24 \pm 0.112 b^{*}$ & $0.15 \pm 0.02 \mathrm{NS}$ & $19.25 \pm 0.41 \mathrm{~b}^{* * *}$ & $2503.9 \mathrm{~b}^{* * * *}$ & $0.43 \pm 0.069 \mathrm{~b}^{* *}$ \\
\hline $\begin{array}{l}\mathrm{Pb}+\mathrm{L} \text {. varians }+\mathrm{P} \text {. } \\
\text { putida }\end{array}$ & $88 \pm 0.651 \mathrm{~b}^{* * *}$ & $7.66 \pm 0.756 b^{* * *}$ & $41.26 \pm 1.995 b^{* * *}$ & $1.36 \pm 0.052 \mathrm{~b}^{* *}$ & $0.21 \pm 0.028 \mathrm{~b}^{* *}$ & $33.83 \pm 0.72 \mathrm{~b}^{* * *}$ & $3631.76 \mathrm{~b}^{* * * *}$ & $0.63 \pm 0.088 \mathrm{~b}^{* * *}$ \\
\hline $\mathrm{Cd}+\mathrm{Pb}$ & $46 \pm 1.036 \mathrm{a}^{* * *}$ & $4.7 \pm 0.389 \mathrm{a}^{* * *}$ & $14.6 \pm 1.384 \mathrm{a}^{* * * *}$ & $0.51 \pm 0.103 \mathrm{a}^{* *}$ & $0.1 \pm 0.03 \mathrm{a}^{* *}$ & $30.76 \pm 0.37 \mathrm{a}^{* * *}$ & $668.38 \mathrm{a}^{* * *}$ & $0.17 \pm 0.035 \mathrm{a}^{* * *}$ \\
\hline $\begin{array}{l}\mathrm{Cd}+\mathrm{Pb}+\mathrm{L} . \\
\text { varians }\end{array}$ & $78 \pm 0.821 \mathrm{~b}^{* * *}$ & $7.03 \pm 0.842 \mathrm{~b}^{* * * *}$ & $38.48 \pm 1.751 \mathrm{~b}^{* * *}$ & $1.22 \pm 0.171 \mathrm{~b}^{* *}$ & $0.15 \pm 0.004 \mathrm{~b}^{*}$ & $25.69 \pm 0.085 \mathrm{~b}^{* * *}$ & $3000 \mathrm{~b}^{* * * *}$ & $0.42 \pm 0.074 \mathrm{~b}^{* * *}$ \\
\hline $\mathrm{Cd}+\mathrm{Pb}+\mathrm{P}$. putida & $64 \pm 0.758 \mathrm{~b}^{* * *}$ & $6.93 \pm 0.204 \mathrm{~b}^{* * *}$ & $35.33 \pm 3.048 \mathrm{~b}^{* * *}$ & $1.07 \pm 0.031 \mathrm{~b}^{* *}$ & $0.12 \pm 0.008 \mathrm{NS}$ & $35.56 \pm 0.49 \mathrm{~b}^{* * *}$ & $2261 \mathrm{~b}^{* * * *}$ & $0.38 \pm 0.064 \mathrm{~b}^{* * *}$ \\
\hline $\begin{array}{l}\mathrm{Cd}+\mathrm{Pb}+\mathrm{L} . \\
\text { varians }+\mathrm{P} . \text { putida }\end{array}$ & $92 \pm 0.651 \mathrm{~b}^{* * *}$ & $7.5 \pm 0.553 \mathrm{~b} * * *$ & $43 \pm 0.612 \mathrm{~b}^{* * *}$ & $1.27 \pm 0.032 \mathrm{~b}^{* *}$ & $0.17 \pm 0.012 \mathrm{~b}^{* *}$ & $39.25 \pm 0.91 \mathrm{~b}^{* * *}$ & $3956 b^{* * * *}$ & $0.49 \pm 0.048 b^{* * *}$ \\
\hline
\end{tabular}

N.B.- Difference between control and respective heavy metal-treated groups are denoted by lower case alphabet 'a'. Lower case alphabet ' $b$ ' indicates difference between heavy metal-treated groups and PGPR inoculated respective heavy metal-treated groups. Asteric mark $(*)$ indicate significance level. 'NS' means not significant.

The above result indicating that the effect of heavy metal stress has been mitigated by the application of PGPR along with the carrier for a sustainable life span of experimental plants. Rajkumar and Freitas (2008) reported that the plant growth parameters were increased by the inoculation of PGPR as compared with noninoculated plant in the heavy-metal stress condition. Whereas, different heavy metal like $\mathrm{Cd}, \mathrm{Pb}$, Ni etc remarkably hampered different growth parameters of different plants (Pal, Chakraborty, and Sengupta 2018; Pal, Mandal, and Sengupta 2019; Pramanik et al. 2016; Pal and Sengupta, 2019). Huang et al., (2016); Gholami et al., (2009) also reported that the application of PGPR increased germination percentage under stressed condition. Both radish and gram plant showed increased chlorophyll content under $\mathrm{Cd}$ and/or $\mathrm{Pb}$ stress condition with PGPR inoculation through carrier than the non-inoculated heavy metal treated condition. Barnawal et.al, (2008) observed similar type of enhancement in chlorophyll content.

PGPRs not only survived within the carrier but also were able to express their potentiality to increase plant growth parameters for a long time. So, the developed carrier can be used for the application of different PGPRs on a large scale for agricultural development.

$\mathrm{Cd}, \mathrm{Pb}$ and $\mathrm{Cd}+\mathrm{Pb}$ significantly $(\mathrm{p}<0.05)$ reduced the vigour index of radish plan by $11.39 \%, 30.37 \%, 45.14 \%$ and $44.05 \%, 4.97 \%, 63.89 \%$ for gram plant Application of PGPR under heavy-metal stress enhanced the vigour index of radish plant by $29.93 \%, 8.30 \%, 3.04 \%$ and for the Gram plant by $47.02 \%, 96.27 \%$, $113.83 \%$ respectively for $L$. varians, $P$. putida and $L$. varians $+P$. putida inoculation as compared with non-inoculated Cadmium treated plants. Similar type of results was noted in other heavy metal combinations. The selected PGPRs applied with developed carrier mixture had a great effect to nullify the adverse effects of heavy metals. The results in this study confirmed the previous results obtained earlier (Aydinalp and Marinova 2009; Pal, Chakraborty, and Sengupta 2018; Pramanik et al. 2016; Rajkumar and Freitas 2008). Moreover, it can be concluded that, the PGPRs within the developed carrier in this study established similar plant growth enhancement in comparison with PGPRs applied with culture media or as water suspension by the previous workers.

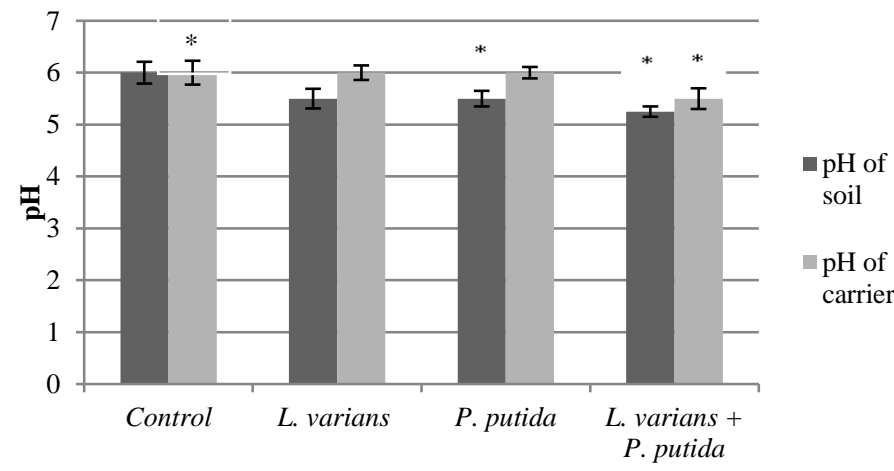

Figure 1 PGPR mediated pH change of soil and carrier.

N.B. Asteric mark above bar $(*)$ indicates significance level between control and respective treatment.

Lysinibacillus varians or Pseudomonas putida inoculation reduced $\mathrm{pH}$ level by $8.3 \%$ than the control soil. Whereas, PGPRs in combination showed $12.5 \%$ reduction of $\mathrm{pH}$. Though, $\mathrm{PGPR}$ inoculation in carrier showed almost similar type of $\mathrm{pH}$ (Figure 1). Lower $\mathrm{pH}$ means increased acidity. Some authors showed that PGPR can produce several kind of organic acid which solubilized insoluble phosphates or potassium. The significant decrease of soil $\mathrm{pH}$ by Plant growthpromoting rhizobacteria (PGPR) reported by Stevenson (2005).

\section{CONCLUSSION}

In the present study two Cadmium and Lead tolerant strain were explored with suitable carrier development. Plant growth promoting ability of Lysinibacillus varians or Pseudomonas putida was previously reported by the authors and in this study they gave similar result while they were grown in a mixture of Wheat Bran, Sesame Bran and Bagasse in 1:1:1 ratio. The carrier ratio was able to provide a good nutrient source for at least 120 days for the PGPRs and showed exceptional CFU count. Lysinibacillus varians strain KUBM17 (accession number MG976681) and Pseudomonas putida strain KUBM18 (accession number MG976684) was not only survived within the carrier but also significantly ( $\mathrm{p}$ 0.05 ) enhanced the plant growth of both radish and gram under cadmium $(\mathrm{Cd})$ and lead $(\mathrm{Pb})$ stress condition throughout their life span. Moreover, the agricultural wastes like Wheat Bran, Sesame Bran and Bagasse can be used for the formulation of a new generation low cost carrier as it contain a good source of carbohydrate and other essential elements for microbial growth. The present study revealed that PGPR could be applied by the carrier as effective bio-fertilizer in almost all soil ecological condition to develop a sustainable agricultural system for the elimination of phyto-toxic effects of cadmium and lead with very little expertise as well as by the farmers themselves.

Author's contribution: AKP conducted the whole experiment, collected the data, performed statistical analysis and written up the whole manuscript. BS supported the experimental works for plant growth parameters. CS hypothesized the paper concept, designed the experiment, supervised throughout the process.

Competing interests: The authors declared that they have no competing interest.

Acknowledgements: The authors would like to acknowledge the financial help from DST-PURSE program to Department of Botany, University of Kalyani, from Department of Science and Technology, GOI, India.

\section{REFFERENCE}

Abdul-Baki, A. A., \& Anderson, J. D. (1973). Vigor determination in soybean seed by multiple criteria 1. Crop science, 13(6), 630-633. https://doi.org/10.2135/cropsci1973.0011183X001300060013x

Alyemeni, M. N., Basahy, A. Y., \& Sher, H. (2011). Physico-chemical analysis and mineral composition of some sesame seeds (Sesamum indicum L.) grown in the Gizan area of Saudi Arabia. Journal of Medicinal Plants Research, 5(2), 270 274.

Ardakani, S. S., Heydari, A., Tayebi, L., \& Mohammadi, M. (2010). Promotion of cotton seedlings growth characteristics by development and use of new bioformulations. International Journal of Botany, 6(2), 95-100.

Awasthi, R., Tewari, R., \& Nayyar, H. (2011). Synergy between plants and Psolubilizing microbes in soils: effects on growth and physiology of crops. International Research Journal of Microbiology, 2(12), 484-503.

Aydinalp, C., \& Marinova, S. (2009). The effects of heavy metals on seed germination and plant growth on alfalfa plant (Medicago sativa). Bulgarian 
Journal of Agricultural Science, 15(4), 347-350. https://doi.org/10.3923/ijb.2010.95.100

Bashan, Y. (1998). Inoculants of plant growth-promoting bacteria for use in agriculture. Biotechnology

https://doi.org/10.1016/S0734-9750(98)00003-2

Bashan, Y., \& de-Bashan, L. E. (2005). Fresh-weight measurements of roots provide inaccurate estimates of the effects of plant growth-promoting bacteria on root growth: a critical examination. Soil Biology and Biochemistry, 37(10), 17951804. https://doi.org/10.1016/j. soilbio.2005.02.013

Choure, K., \& Dubey, R. C. (2012). Development of plant growth promoting microbial consortium based on interaction studies to reduce wilt incidence in Cajanus cajan L. var. Manak. World Journal of Agricultural Sciences, 8(1), 118128

Dotaniya, M. L., Datta, S. C., Biswas, D. R., Dotaniya, C. K., Meena, B. L., Rajendiran, S., ... \& Lata, M. (2016). Use of sugarcane industrial by-products for improving sugarcane productivity and soil health. International Journal of Recycling of Organic Waste in Agriculture, 5(3), 185194.https://doi.org/10.1007/s40093-016-0132-8

Fardet, A. (2010). New hypotheses for the health-protective mechanisms of wholegrain cereals: what is beyond fibre?. Nutrition research reviews, 23(1), 65-134. https://doi.org/10.1017/S0954422410000041

Faruk, M. I., Rahman, M. L., Islam, M. N., Rahman, M. M., \& Rahman, M. A. (2014). Assessment of Carrier Materials to Formulate Trichoderma Harzianum Bio-Fungicide for Controlling Foot and Root Rot Disease of Brinjal in Seed Bed (No. RESEARCH). https://doi.org/10.3329/bjar.v39i2.20415

Fuentes-Ramirez, L.E. and Caballero-Mellado, J., (2005). PGPR: Biocontrol and Biofertilization. Springer Publisher, Netherlands.

Gholami, A., Shahsavani, S., \& Nezarat, S. (2009). The effect of plant growth promoting rhizobacteria (PGPR) on germination, seedling growth and yield of maize. World Academy of Science, Engineering and Technology, 49, 19-24.

Jackson, M. L. (1967). Soil Chemical Analysis (Ed.) Prentice Hall of India Private Limited. New Delhi.

Marchiol, L., Assolari, S., Sacco, P., \& Zerbi, G. (2004). Phytoextraction of heavy metals by canola (Brassica napus) and radish (Raphanus sativus) grown on multicontaminated soil. Environmental Pollution, 132(1), 21-27. https://doi.org/10.1016/j.envpol.2004.04.001

Ningthoujam, D. S., Sanasam, S., \& Nimaichand, S. (2009). Screening of actinomycete isolates from niche habitats in Manipur for antibiotic activity. Am. $J$ Biochem. Biotechnol, 5(4), 221-225. https://doi.org/10.3844/ajbbsp.2009.221.225 Pal, A. K., \& Sengupta, C. (2019). Isolation of cadmium and lead tolerant plant growth promoting rhizobacteria: Lysinibacillus varians and Pseudomonas putida from Indian Agricultural Soil. Soil and Sediment Contamination: An International Journal, 28(7), 601-629. https://doi.org/10.1080/15320383.2019.1637398

Pal, A. K., Chakraborty, A., \& Sengupta, C. (2018). Differential effects of plant growth promoting rhizobacteria on chilli (Capsicum annuum L.) seedling under cadmium and lead stress. Plant Science Today, 5(4), 182-190. https://doi.org/10.14719/pst.2018.5.4.419

Pal, A. K., Mandal, S., \& Sengupta, C. (2019). Exploitation of IAA Producing PGPR on mustard (Brassica nigra L.) seedling growth under cadmium stress condition in comparison with exogenous IAA application. Plant Science Today, 6(1), 22-30. https://doi.org/10.14719/pst.2019.6.1.440

Porra, R. J., Thompson, W. A., \& Kriedemann, P. E. (1989). Determination of accurate extinction coefficients and simultaneous equations for assaying chlorophylls a and b extracted with four different solvents: verification of the concentration of chlorophyll standards by atomic absorption spectroscopy. Biochimica et Biophysica Acta (BBA)-Bioenergetics, 975(3), 384394. https://doi.org/10.1016/S0005-2728(89)80347-0

Pramanik, K., Ghosh, P. K., Ghosh, A., Sarkar, A., \& Maiti, T. K. (2016) Characterization of PGP traits of a hexavalent chromium resistant Raoultella sp. isolated from the rice field near industrial sewage of Burdwan District, WB, India. Soil and Sediment Contamination: An International Journal, 25(3), 313331. https://doi.org/10.1080/15320383.2016.1137861

Prikryl, Z., \& Vancura, V. (1980). Root exudates of plants, 6: wheat root exudation as dependent on growth, concentration gradient of exudates and the presence of bacteria. Plant and Soil (Netherlands). https://doi.org/10.1007/BF02139643

Rajkumar, M., \& Freitas, H. (2008). Effects of inoculation of plant-growth promoting bacteria on $\mathrm{Ni}$ uptake by Indian mustard. Bioresource Technology, 99(9), 3491-3498. https://doi.org/10.1016/j.biortech.2007.07.046

Ravindran, R., \& Jaiswal, A. K. (2016). Exploitation of food industry waste for high-value products. Trends in Biotechnology, 34(1), 58-69. https://doi.org/10.1016/j.tibtech.2015.10.008

Samra, J. S., Sharma, P. D., \& Kumar, V. (2003). The use of fly ash in agriculture in India. In Central Board of Irrigation and Power-3rd International ConferenceFly Ash Utilization \& Disposal (pp. 19-21).

Srivastava, A., \& Chhonkar, P. K. (2000). Effect of flyash on uptake of phosphorus, potassium and sulphur by Sudan grass and oats grown on an acid soil. Journal of the Indian Society of Soil Science, 48(4), 850-853.

Zouari, R., Besbes, S., Ellouze-Chaabouni, S., \& Ghribi-Aydi, D. (2016). Cookies from composite wheat-sesame peels flours: Dough quality and effect of Bacillus subtilis SPB1 biosurfactant addition. Food chemistry, 194, 758-769. https://doi.org/10.1016/j.foodchem.2015.08.064 